\title{
001
}

\section{RIEMERGENZA DI SALMONELLA SIEROTIPO NAPOLI IN ITALIA E IN EUROPA}

Dionisi A.M., Galetta P., Owczarek S., Filetici E., Benedetti I., Luzzi I. e i partcipanti alla rete Enter-Net.

Istituto Superiore di Sanità,

viale Regina Elena 299,00161 Roma

Salmonella Napoli ha rappresentato nel corso degli anni un sierotipo piuttosto raro. Negli ultimi quattro anni tuttavia il sistema EnterNet Italia ha registrato un aumento di questo sierotipo in casi di infezione e da fonti ambientali (acqua superficiale).

La maggior parte degli isolamenti è stata registrata in Lombardia in particolare nella provincia di Varese. Un incremento nella frequenza di isolamento è stata osservato anche in altri Paesi europei ed in particolare in Svizzera in una zona al confine con la Lombardia bagnata dal Lago Maggiore. Sia in Italia che i Europa si è osservato un andamento stagionale con un picco di isolamenti nei mesi di luglio-ottobre.

Al fine di correlare i ceppi isolati da casi di infezione e da campioni ambientali e di correlare gli isolamenti italiani con quelli Svizzeri, è stata effettuata un'analisi molecolare mediante elettroforesi pulsata (PFGE).

La PFGE è stata eseguita secondo un protocollo standardizzato a livello europeo (progetto Salmgene). I profili ottenuti mediante PFGE sono stati analizzati utilizzando il software Bionumerics ed è stata eseguita una "cluster analysis" per raggruppare i ceppi analizzati in base alle omologie.

I ceppi di S. Napoli inviati dai laboratori partecipanti alla rete Enter-net sono stati analizzati mediante PFGE.

La "cluster analysis" ha evidenziato una stretta correlazione tra i ceppi umani ed ambientali provenienti dalla Lombardia. L'analisi ha inoltre messo in evidenza una stretta omologia tra $\mathrm{i}$ ceppi isolati in provincia di Varese e i ceppi isolati in Svizzera. Una debole correlazione genetica è emersa tra $\mathrm{i}$ ceppi isolati in altre regioni italiane.

La PFGE rappresenta un utile strumento di epidemiologia molecolare e può essere utilizzato per monitorare e descrivere la circolazione di particolari cloni nelle varie fonti e serbatoi. Nel caso specifico ha consentito di correlare ceppi isolati da fonti diverse e da diverse aree geografiche. 